\title{
Joint Vehicle Trajectory and Model Parameter Estimation using Road Side Sensors
}

\author{
Roland Hostettler, Wolfgang Birk, and Magnus Lundberg Nordenvaad
}

This is a post-print of a paper published in IEEE Sensors Journal. When citing this work, you must always cite the original article:

R. Hostettler, W. Birk, and M. Lundberg Nordenvaad, "Joint vehicle trajectory and model parameter estimation using road side sensors," Sensors Journal, IEEE, vol. 15, no. 9, pp. 50755086, Sept 2015

\section{DOI:}

10.1109/JSEN.2015.2432748

\section{Copyright:}

(c) 2015 IEEE. Personal use of this material is permitted. Permission from IEEE must be obtained for all other uses, in any current or future media, including reprinting/republishing this material for advertising or promotional purposes, creating new collective works, for resale or redistribution to servers or lists, or reuse of any copyrighted component of this work in other works. 


\title{
Joint Vehicle Trajectory and Model Parameter Estimation using Road Side Sensors
}

\author{
Roland Hostettler, Member, IEEE, Wolfgang Birk, Member, IEEE, Magnus Lundberg Nordenvaad
}

\begin{abstract}
This article shows how a particle smoother based system identification method can be applied for estimating the trajectory of road vehicles. As sensors, a combination of an accelerometer measuring the road surface vibrations and a magnetometer measuring magnetic disturbances mounted on the side of the road are considered. First, sensor models describing the measurements of the two sensors are introduced. It is shown that these depend on unknown, static parameters that have to be considered in the estimation. Second, the sensor models are combined with a two-dimensional constant velocity motion model. Third, the system identification algorithm is introduced which iteratively runs a Rao-Blackwellized particle smoother to estimate the vehicle trajectory followed by an expectation-maximization step to estimate the parameters. Finally, the method is applied to both simulation and measurement data. It is found that the method works well in general and some issues when real data is considered are identified as future work.
\end{abstract}

Index Terms-State Estimation, Parameter Estimation, Accelerometers, Magnetometers

\section{INTRODUCTION}

Estimating target trajectories is of interest in many different applications such as air traffic control, tracking marine vessels, or estimating the driving path of ground-borne vehicles [2]. This is often achieved by combining a motion model for the target with a sensor model that describes the measured signal as a function of the motion. The motion model describes the motion in a way suitable for the application, for example using Cartesian or polar coordinates and it is possible to convert the problem between the different representations [3]. The sensor model is given by the sensing technique used and it is not uncommon that it depends on a set of unknown variables parameterizing the model [4].

In this article, estimating the vehicle trajectory using a combination of an accelerometer and a magnetometer, both mounted in a single sensor unit and installed on the road surface, is considered. The accelerometer measures the vibrations on the road surface caused by a vehicle passing the sensor. The magnetometer on the other hand measures the field vector of local magnetic disturbances caused by the metallic mass of a vehicle. In this setting, both sensor models are nonlinear and depend on a set of static, material-specific parameters which complicate the tracking problem.

Target tracking using both kinds of sensors has been considered individually before. For example, it has been shown

This work has been supported by GEVEKO ITS A/S

R. Hostettler and W. Birk are with the Division of Signals and Systems, Luleå University of Technology, Luleå, Sweden (e-mail: roland.hostettler@1tu.se).

M. Lundberg Nordenvaad is with the Division of Systems and Control, Uppsala University, Uppsala, Sweden. that it is possible to detect and track activities of heavy military vehicles by measuring ground vibrations [5]. In [6], it was shown that it is feasible to use an extended Kalman filter for tracking road vehicles using accelerometers. Problems arose regarding the material parameters which were treated as tuning parameters. Similarly, magnetometers have been used for tracking in different contexts such as underwater object tracking or road vehicle tracking [7]-[10]. Magnetometers, in combination with odometry, have also been shown to be a viable option for indoor simultaneous localization and mapping (SLAM) for robots [11], [12] as well as humans [12], [13]. Finally, location tracking by using magnetometers and MEMS inertial measurements based on a priori known maps was proposed in [14]. However, fusion of the accelerometer and magnetometers for vehicle trajectory estimation under the above mentioned circumstances is a novel approach.

Estimating both, the system states (in this case the target trajectory) and model parameters is a well-known problem that can be approached in many different ways. One popular method that has proven very efficient, especially for linear systems, is to augment the state vector with the unknown parameters, treating them as extra states [15]. Then, the parameters are estimated on-line together with the original states, for example by using a Kalman-Bucy filter. This approach can, however, be problematic in nonlinear systems where approximative methods such as particle filters have to be employed. In such a situation, special attention has to be paid if the state augmentation approach is chosen since the static parameters might lead to particle impoverishment [16], [17]. Instead of augmenting the state vector which yields continuously updated parameter estimates, one can also resort to on-line methods that yield point estimates. One such approach was introduced in [18] where Markov chain Monte Carlo simulation in conjunction with fixed-lag smoothing was used to obtain the estimates. Furthermore, [19] showed how to use sequential Monte Carlo methods together with a gradient search based on-line expectation maximization.

If on-line estimation is not a requirement, for example when auto-tuning or initializing a measurement system, one can instead resort to off-line approaches. In [20], a particle filter for batch estimation of parameters only was introduced. Other methods use gradient based maximum likelihood estimation, see for example [21]. Yet another approach is to separate the task into two mutually coupled problems [15]. When using such an approach, one iteratively first estimates the states using a smoother (given feasible candidates of the parameters), followed by an expectation maximization step where improved estimates of the parameters are found based on the new state 
estimates [22], [23].

The contribution of this work is twofold. First, it is shown how the nonlinear sensor fusion problem described above can be formulated in terms of a dynamic motion model and two sensor models. Second, the expectation-maximization based system identification algorithm introduced in [23] is applied for estimating the vehicle trajectory and model parameters. While not being an on-line method, this approach has proven to be a good choice in such situations and is the one favored here as real-time operation is not a requirement. For obtaining smoothed state estimates, a forward filtering backward simulation Rao-Blackwellized particle smoother based on [24], [25] and [26] is proposed. This is motivated by the fact that both sensor models are non-linear functions of the states which requires either the usage of, for example extended or unscented Kalman smoothers, or particle smoothing methods where it has been shown that the latter have superior performance in general, see for example [27]. Also, there are a total of 6 unknown model parameters to be estimated which would considerably blow up the state vector if the state vector augmentation approach were chosen. Finally, the method is also compared to the case where the joint smoothing density is approximated using an unscented Rauch-Tung-Striebel smoother (URTSS) [28] as introduced in [29]. Approximating the posterior using an URTSS is chosen for comparison as this approach can be deemed the most comparable approach to the proposed method, see above.

The remainder of this article is organized as follows. The motion and sensing models governing the problem are introduced in Section II, followed by the description of the method in Section III. The performance under ideal conditions is verified using Monte Carlo simulations in Section IV. Finally, the method is applied to real measurement data in Section V and concluding remarks are given in Section VI.

\section{MODEL}

\section{A. Motion Model}

The motion of a target can be described by using different models, depending on the parametrization of the coordinate system and the type of motion of interest. For a vehicle moving along a road, a Cartesian coordinate system with the $\mathrm{X}$-coordinate $r^{x}$ in longitudinal and the $\mathrm{y}$-coordinate $r^{y}$ across the road (lateral) is a reasonable choice. Then, using the discretized two-dimensional constant velocity motion model [3], [30], the motion of a vehicle is described by

$$
x_{t+1}=\underbrace{\left[\begin{array}{cccc}
1 & 0 & T_{s} & 0 \\
0 & 1 & 0 & T_{s} \\
0 & 0 & 1 & 0 \\
0 & 0 & 0 & 1
\end{array}\right]}_{\triangleq A} x_{t}+\underbrace{\left[\begin{array}{cccc}
\frac{T_{s}^{2}}{2} & 0 & 0 & 0 \\
0 & \frac{T_{s}^{2}}{2} & 0 & 0 \\
0 & 0 & T_{s} & 0 \\
0 & 0 & 0 & T_{s}
\end{array}\right]}_{\triangleq B} v_{t}
$$

where the state vector is $x_{t}=\left[\begin{array}{llll}r_{t}^{x} & r_{t}^{y} & \dot{r}_{t}^{x} & \dot{r}_{t}^{y}\end{array}\right]^{T}$, the subscript $t=1, \ldots, T$ denotes the discrete time index, and $T_{s}$ is the sampling time. The process noise $v_{t}$ is assumed to be of the form

$$
p\left(v_{t}\right)=\mathcal{N}\left(v_{t} ; 0, Q\right)
$$

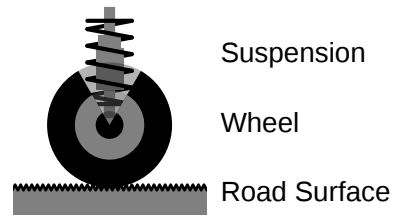

Fig. 1. Illustration of the interaction between a vehicle wheel and the road surface.

with

$$
Q=\left[\begin{array}{cccc}
\sigma_{x}^{2} & 0 & 0 & 0 \\
0 & \sigma_{y}^{2} & 0 & 0 \\
0 & 0 & \sigma_{x}^{2} & 0 \\
0 & 0 & 0 & \sigma_{y}^{2}
\end{array}\right]
$$

Furthermore, the initial state $x_{1}$ is assumed to be distributed as

$$
p\left(x_{1}\right)=\mathcal{N}\left(x_{1} ; \mu_{x_{1}}, C_{x_{1}}\right)
$$

Finally, the location of the target at time $t$ is given by the vector

$$
r_{t}=\left[\begin{array}{c}
r_{t}^{x} \\
r_{t}^{y} \\
0
\end{array}\right]
$$

The position in z-direction $r_{t}^{z}$ is assumed to be zero since the vehicle and sensor are approximately in the same plane.

\section{B. Accelerometer Sensor Model}

It has been shown [31] that vibrations normal to the road surface measured by an accelerometer can be modeled as a linear system of the form

$$
Z_{a}(\omega)=H(r, \omega) U(\omega)+E_{a}(\omega)
$$

where $\omega$ is the circular frequency, $U(\omega)$ is the excitation, $Z_{a}(\omega)$ the measured vibrations, $E_{a}(\omega)$ the measurement noise, and $r$ is the vector pointing from the sensor to the source. The transfer function $H(r, \omega)$ is given by

$$
H\left(r_{t}, \omega\right)=\alpha(\omega) \mathcal{H}_{0}^{(1)}(-k(\omega)|r|)
$$

where $\alpha(\omega)$ is a frequency dependent, complex gain, $\mathcal{H}_{0}^{(1)}(x)$ is the Hankel function of the first kind of order zero [32], and

$$
k=\frac{\omega}{c_{p}(\omega)}-i \eta(\omega)
$$

is the complex wavenumber with $c_{p}$ the frequency-dependent phase velocity and $\eta$ an attenuation constant.

Assuming that the parameters $\alpha(\omega) \approx \alpha$ and $\eta(\omega) \approx \eta$ are approximately constant for a limited frequency band of interest and that $c_{p}(\omega) \gg \omega,(7)$ can be approximated as

$$
H(r, \omega) \approx \kappa i \mathcal{H}_{0}^{(1)}(i \eta|r|)
$$

where $\kappa \triangleq|\alpha|$ and the imaginary unit $i$ ensures a real-valued function.

Note that (8) is independent of $\omega$ and hence, taking the inverse Fourier transform of the measured signal (6) yields

$$
z_{a, t} \approx \kappa i \mathcal{H}_{0}^{(1)}(i \eta|r|) u_{t}+e_{a, t} .
$$


For the source, note that the excitation $u_{t}$ is caused by the interaction of the vehicle's $2 P$ wheels (where $P$ is the number of axles) and the road (Fig. 1). This interaction results in a normal force which depends on the road surface profile as well as vehicle properties such as the characteristics of the suspension and the vehicle weight. The road surface profile is of interest in different applications including riding comfort assessment or road maintenance [33]-[35]. It is commonly modeled as a random process and characterized by a power spectral density (PSD) such as specified by the ISO 8608 standard [36]. It is thus assumed that the excitation can be modeled as a zero mean, Gaussian random variable in the time domain, that is,

$$
u_{t} \sim \mathcal{N}(0,1)
$$

Considering all $P$ axles and $r_{p, t}$ being the position of the $p^{\text {th }}$ axle at time $t$, the measurement model becomes

$$
y_{a, t}=\sum_{p=1}^{P} \kappa i \mathcal{H}_{0}^{(1)}\left(i \eta\left|r_{p, t}\right|\right) u_{t}+e_{a, t}
$$

Without loss of generality, it is assumed that $P=2$ for the remainder of this paper, which represents the largest group of vehicles including passenger cars and light trucks. Thus, the measurement model becomes

$$
y_{a, t}=\underbrace{\kappa i\left(\mathcal{H}_{0}^{(1)}\left(i \eta\left|r_{1, t}\right|\right)+\mathcal{H}_{0}^{(1)}\left(i \eta\left|r_{2, t}\right|\right)\right)}_{\triangleq h_{a}\left(x_{t}\right)} u_{t}+e_{a, t}
$$

The locations of the axles $r_{1, t}$ and $r_{2, t}$ can be expressed by using the wheelbase $l$ as

$$
r_{1, t}=r_{t}+\left[\begin{array}{c}
l / 2 \\
0 \\
0
\end{array}\right] \quad \text { and } \quad r_{2, t}=r_{t}-\left[\begin{array}{c}
l / 2 \\
0 \\
0
\end{array}\right]
$$

The measurement noise in the sensor $e_{a, t}$ is due to thermal noise, sensor imperfections, etc. and is modeled as white Gaussian noise with PDF

$$
p\left(e_{a, t}\right)=\mathcal{N}\left(e_{a, t} ; 0, \sigma_{a}^{2}\right) .
$$

\section{Magnetometer Sensor Model}

Target tracking using magnetometer sensors has been explored quite extensively [8]-[10]. It has been shown that the measured magnetic disturbance caused by a vehicle passing a magnetometer can be modeled as a dipole or, if the magnetic mass of the vehicle has a larger geometry, as a series of dipoles [10]. The magnetic disturbance caused by a dipole is given by

$$
y_{m, t}=\underbrace{\frac{3\left(r_{t}^{T} m\right) r_{t}-\left|r_{t}\right|^{2} m}{\left|r_{t}\right|^{5}}}_{\triangleq h_{m}\left(x_{t}\right)}+e_{m, t}
$$

where $y_{m, t}$ denotes the measured magnetic disturbance, a $3 \times 1$ vector, and $m=\left[\begin{array}{lll}m^{x} & m^{y} & m^{z}\end{array}\right]^{T}$ is the magnetic dipole moment of the object.
Even for the magnetometer, the measurement noise is assumed to be white Gaussian noise according to

$$
p\left(e_{m, t}\right)=\mathcal{N}\left(e_{m, t} ; 0, C_{m}\right)
$$

with

$$
C_{m}=\left[\begin{array}{ccc}
\sigma_{m, x}^{2} & 0 & 0 \\
0 & \sigma_{m, y}^{2} & 0 \\
0 & 0 & \sigma_{m, z}^{2}
\end{array}\right]
$$

Note that the amount of noise can vary between the different components which is reflected in the individual $\sigma_{m, x}^{2}, \sigma_{m, y}^{2}$, and $\sigma_{m, z}^{2}$, depending on the sensor configuration.

\section{Complete Model}

Summarizing the models introduced above, the following general state-space model is obtained

$$
\begin{aligned}
x_{t+1} & =A x_{t}+B v_{t} \\
y_{t} & =\left[\begin{array}{c}
h_{a}\left(x_{t}\right) u_{t} \\
h_{m}\left(x_{t}\right)
\end{array}\right]+\left[\begin{array}{c}
e_{a, t} \\
e_{m, t}
\end{array}\right]
\end{aligned}
$$

Theoretically, (18) could now be used for tracking directly if all the parameters were known. However, closer inspection shows two things. First, both measurement models depend on unknown parameters. For the accelerometer, the material parameters $\kappa$ and $\eta$ as well as the wheelbase $l$ are unknown. For the magnetometer, the magnetic moment $m$ is unknown. This yields the parameter vector

$$
\theta=\left[\begin{array}{llll}
\kappa & \eta & l & m^{T}
\end{array}\right]^{T}
$$

and $\theta$ is added as a parametrization to the measurement functions, that is, $h_{a}\left(x_{t} ; \theta\right)$ and $h_{m}\left(x_{t} ; \theta\right)$.

Second, the structure of (18) makes it possible to split the state vector into non-linear and linear states which will be helpful when applying the particle smoothing algorithm. The only states appearing in the non-linear measurement functions are the positions $r_{t}^{x}$ and $r_{t}^{y}$ whereas $\dot{r}_{t}^{x}$ and $\dot{r}_{t}^{y}$ do not appear in the measurement equations. Hence, the state vector can be divided into linear and non-linear states as follows

$$
x_{t}^{n}=\left[\begin{array}{c}
r_{t}^{x} \\
r_{t}^{y}
\end{array}\right] \quad \text { and } \quad x_{t}^{l}=\left[\begin{array}{c}
\dot{r}_{t}^{x} \\
\dot{r}_{t}^{y}
\end{array}\right]
$$

where the superscript $n$ and $l$ denote the non-linear and linear state variables, respectively.

The model (18) can then be rewritten as

$$
\begin{aligned}
x_{t+1}^{n} & =F^{n} x_{t}^{n}+A^{n} x_{t}^{l}+B^{n} v_{t} \\
x_{t+1}^{l} & =A^{l} x_{t}^{l}+B^{l} v_{t} \\
y_{t} & =\left[\begin{array}{c}
h_{a}\left(x_{t}^{n} ; \theta\right) u_{t} \\
h_{m}\left(x_{t}^{n} ; \theta\right)
\end{array}\right]+\left[\begin{array}{c}
e_{a, t} \\
e_{m, t}
\end{array}\right]
\end{aligned}
$$


where

$$
\begin{aligned}
& F^{n}=\left[\begin{array}{ll}
1 & 0 \\
0 & 1
\end{array}\right]=I_{2}, \\
& A^{n}=\left[\begin{array}{cc}
T_{s} & 0 \\
0 & T_{s}
\end{array}\right]=T_{s} I_{2}, \\
& A^{l}=\left[\begin{array}{ll}
1 & 0 \\
0 & 1
\end{array}\right]=I_{2}, \\
& B^{n}=\left[\begin{array}{cccc}
\frac{T_{s}^{2}}{2} & 0 & 0 & 0 \\
0 & \frac{T_{s}^{2}}{2} & 0 & 0
\end{array}\right]=\left[\begin{array}{cc}
\frac{T_{s}^{2}}{2} I_{2} & 0
\end{array}\right]
\end{aligned}
$$

and

$$
B^{l}=\left[\begin{array}{cccc}
0 & 0 & T_{s} & 0 \\
0 & 0 & 0 & T_{s}
\end{array}\right]=\left[\begin{array}{ll}
0 & T_{s} I_{2}
\end{array}\right] .
$$

Finally, the model can be written in terms of probability density functions. The state transition probability density is given through the state dynamics (21a)-(21b) and is

$$
\begin{aligned}
& p\left(x_{t+1} \mid x_{t}\right) \\
& \quad=\mathcal{N}\left(\left[\begin{array}{c}
x_{t+1}^{n} \\
x_{t+1}^{l}
\end{array}\right] ;\left[\begin{array}{cc}
F^{n} & A^{n} \\
0 & A^{l}
\end{array}\right]\left[\begin{array}{c}
x_{t}^{n} \\
x_{t}^{l}
\end{array}\right],\left[\begin{array}{c}
B^{n} \\
B^{l}
\end{array}\right] Q\left[\begin{array}{c}
B^{n} \\
B^{l}
\end{array}\right]^{T}\right) .
\end{aligned}
$$

The likelihood is given through the measurement equations (21c). The measurement noises of the accelerometer and magnetometer are assumed to be uncorrelated and hence, the joint likelihood is the product of the individual likelihoods and given by

$$
\begin{aligned}
p\left(y_{t} \mid x_{t} ; \theta\right)= & p\left(y_{a, t} \mid x_{t} ; \theta\right) p\left(y_{m, t} \mid x_{t} ; \theta\right) \\
= & \mathcal{N}\left(y_{a, t} ; 0, h_{a}\left(x_{t} ; \theta\right)^{2}+\sigma_{a}^{2}\right) \\
& \times \mathcal{N}\left(y_{m, t} ; h_{m}\left(x_{t} ; \theta\right), C_{m}\right) .
\end{aligned}
$$

\section{METHOD}

Given the nonlinear estimation problem (21) introduced in Section II, it will now be shown how the particle system identficiation algorithm introduced in [23] can be applied to this problem. As a result, both the state vector $x_{t}$ and the unknown model parameters $\theta$ can be estimated. Note that the algorithms introduced here are simply stated for completeness but are described and derived in the respective references.

\section{A. Particle System Identification}

The system identification algorithm as proposed by [23] is summarized in Algorithm 1. It uses sequential Monte Carlo methods to obtain state estimates given candidate parameters $\hat{\theta}_{k}$ and then finds an improved estimate $\hat{\theta}_{k+1}$ using expectation-maximization (EM). Note that even though the EM algorithm in general guarantees an increase in likelihood for $\theta$ for each iteration, this is only asymptotically true when using particle Monte Carlo methods (as the number of particles goes to infinity), see [23] for details.

Algorithm 1 (Particle EM System Identification).

1) Set $k \leftarrow 0$ and choose a feasible starting point $\hat{\theta}_{0}$.

2) Expectation step:

a) Run a particle smoother given a candidate $\hat{\theta}_{k}$ and store the filtered particles $\tilde{x}_{t \mid t}^{(n)}$ and their weights $w_{t \mid t}^{(n)}$ and the smoothed particles $\tilde{x}_{t \mid T}^{(m)}$ and their

weights $w_{t \mid T}^{(m)}$.

b) Estimate the expected log-likelihood

$$
\hat{Q}_{M}\left(\theta, \hat{\theta}_{k}\right)=\hat{J}_{1}+\hat{J}_{2}+\hat{J}_{3}
$$

3) Maximization step:

$$
\hat{\theta}_{k+1}=\underset{\theta}{\operatorname{argmax}} \hat{Q}_{M}\left(\theta, \hat{\theta}_{k}\right)
$$

4) If $Q\left(\hat{\theta}_{k+1}, \hat{\theta}_{k}\right)-Q\left(\hat{\theta}_{k}, \hat{\theta}_{k}\right)>\epsilon$ then set $k \leftarrow k+1$ and return to step 2 , otherwise terminate.

Step 2a) in Algorithm 1 requires to run a particle smoother in order to obtain the $n=1, \ldots, M_{F}$ filtered and $m=$ $1, \ldots, M_{S}$ smoothed particles and their weights. These can be obtained from any particle smoothing algorithm, such as [25] or [37]. Furthermore, the terms $\hat{J}_{1}, \hat{J}_{2}$, and $\hat{J}_{3}$ in (23) are given by [23]

$$
\begin{aligned}
& \hat{J}_{1}=\sum_{m=1}^{M_{S}} w_{t \mid T}^{(m)} \log \left(p\left(\tilde{x}_{1 \mid T}^{(m)}\right)\right) \\
& \hat{J}_{2}=\sum_{t=1}^{T-1} \sum_{m=1}^{M_{F}} \sum_{n=1}^{M_{S}} w_{t \mid T}^{(m, n)} \log \left(p\left(\tilde{x}_{t+1 \mid T}^{(n)} \mid \tilde{x}_{t \mid t}^{(m)}\right)\right) \\
& \hat{J}_{3}=\sum_{t=1}^{T} \sum_{m=1}^{M_{S}} w_{t \mid T}^{(m)} \log \left(p\left(y_{t} \mid \tilde{x}_{t \mid T}^{(m)} ; \theta\right)\right)
\end{aligned}
$$

where

$$
w_{t \mid T}^{(m, n)}=\frac{w_{t \mid t}^{(m)} w_{t+1 \mid T}^{(n)} p\left(\tilde{x}_{t+1 \mid T}^{(n)} \mid \tilde{x}_{t \mid t}^{(m)}\right)}{\sum_{l=1}^{M_{F}} w_{t \mid t}^{(l)} p\left(\tilde{x}_{t+1 \mid T}^{(n)} \mid \tilde{x}_{t \mid t}^{(l)}\right)} .
$$

Finally, Step 3) requires to maximize the expected loglikelihood $\hat{Q}_{M}$ to obtain an improved estimate $\hat{\theta}_{k+1}$ of the parameters $\theta$. Steps 2a) and 3) are addressed more thoroughly in the next sections.

\section{B. State Smoothing}

As indicated, Step 2a) in Algorithm 1 requires a particle smoother in order to compute an approximation of the marginal smoothing density [37]. Since the model (21) is a conditionally Gaussian linear state-space system, the joint smoothing density can be written as

$$
p\left(x_{1: T}^{l}, x_{1: T}^{n} \mid y_{1: T}\right)=p\left(x_{1: T}^{l} \mid x_{1: T}^{n}, y_{1: T}\right) p\left(x_{1: T}^{n} \mid y_{1: T}\right)
$$

and only the nonlinear states have to be targeted by the particle smoother. The linear states in turn can be computed conditioned on the nonlinear states, for example using a RauchTung-Striebel smoother [38]. Hence, a Rao-Blackwellized particle smoother only targeting the smoothing density

$$
p\left(x_{1: T}^{n} \mid y_{1: T}\right)
$$

is required. This has the advantage that the dimension of the states that are estimated using Monte Carlo methods is reduced and hence, fewer particles are required. The marginal smoothing density $p\left(x_{t} \mid y_{1: T}\right)$ required in Algorithm 1 is then obtained by marginalizing the joint smoothing density as

$$
p\left(x_{t} \mid y_{1: T}\right)=\int_{-\infty}^{\infty} p\left(x_{1: T} \mid y_{1: T}\right) d x_{1: t-1} d x_{t+1: T} .
$$


A Rao-Blackwellized forward filtering backward simulation (RB-FFBSi) smoothing algorithm targeting (28) and suitable for this problem was introduced in [26]. Assume that one is given an approximation of the partial backward state trajectory $\tilde{x}_{t+1: T \mid T}^{n,(m)}$. Then, one would like to extend this trajectory to time $t$ by using the particles $\tilde{x}_{t \mid t}^{n,(n)}$ from a Rao-Blackwellized particle filter (RB-PF). The RB-FFBSi algorithm is based on calculating the smoothed particle weight

$$
w_{t \mid T}^{(n, m)} \propto w_{t \mid t}^{(n)} Z_{t}^{(n, m)}\left|\Lambda_{t}^{(n, m)}\right|^{-1 / 2} \exp \left(-\frac{1}{2} \eta_{t}^{(n, m)}\right)
$$

given that the $m$-th trajectory is extended using the $n$-th particle. $w_{t \mid t}^{(n)}$ is the filtering weight for the $n$-th particle obtained from the RB-PF. Equation (30) is obtained from the Rao-Blackwellization of the smoothing density with respect to the linear states, see [26] for the derivation. The expressions for the quantities $Z_{t}^{(n, m)}, \Lambda_{t}^{(n, m)}$, and $\eta_{t}^{(n, m)}$ are given in the Appendix.

For each trajectory $\tilde{x}_{t+1: T \mid T}^{n,(m)}\left(m=1, \ldots, M_{S}\right)$ that one would like to extend to time $t$, one could now calculate the weight (30) for each filter particle $\tilde{x}_{t \mid t}^{n,(n)}\left(n=1, \ldots, M_{F}\right)$ and then take a sample from the categorical distribution $\mathcal{C}\left(\left\{w_{t \mid T}^{(n, m)}\right\}_{n=1}^{M_{F}}\right)$ in order to choose a sample for the extension. However, this is highly inefficient, resulting in a smoother that scales with $\mathcal{O}\left(T \cdot M_{S} \cdot M_{F}\right)$. Instead, we chose to combine the above Rao-Blackwellized smoothing strategy with the rejection sampling based smoother proposed in [25] which asymptotically (as $M_{S} \rightarrow \infty$ ) scales with $\mathcal{O}\left(T \cdot M_{S}\right.$ ). Further, in order to avoid getting trapped inside the rejection sampling phase of the smoother, we also implement adaptive stopping as proposed in [39]. Finally, it is very important to point out that the smoothed weight in (30) is only used for the rejection sampling (Step 7f)) and categorical sampling (Step 8c)) stages but not as the weights of the smoothed particles. This is due to the backward-simulation strategy that creates samples that are approximately distributed according to the smoothing density with smoothed weights $w_{t \mid T}^{(m)}=1 / M_{S}$ [15]. The final particle smoothing algorithm is illustrated in Algorithm 2.

Algorithm 2 (Rao-Blackwellized Rejection Sampling Particle Smoother with Adaptive Stopping).

1) Run a Rao-Blackwellized particle filter and store the particles $\tilde{x}_{t \mid t}^{n,(n)}$, their weights $w_{t \mid t}^{(n)}$, the conditionally linear states $\tilde{x}_{t \mid t}^{l,(n)}$, and covariances $P_{t \mid t}^{(n)}$

2) Set $t \leftarrow T$

3) Sample $j \sim \mathcal{C}\left(\left\{w_{T \mid T}^{(n)}\right\}_{n=1}^{M_{F}}\right)$ and set $\tilde{x}_{T \mid T}^{n,(m)}=\tilde{x}_{T \mid T}^{n,(j)}$

4) Initialize $\hat{\Omega}_{T}^{(m)}$ and $\hat{\lambda}_{T}^{(m)}$ according to (49)

5) Set $t \leftarrow t-1$

6) Set $L \leftarrow\left\{1, \ldots, M_{S}\right\}$ and $k \leftarrow 0$

7) While $L$ not empty and $k<K$, then, for each $m \in L$

a) Sample $j \sim \mathcal{C}\left(\left\{w_{t \mid t}^{(n)}\right\}_{n=1}^{M_{F}}\right)$

b) Calculate $Z_{t}^{(j, m)}, \Omega_{t}^{(j, m)}$, and $\lambda_{t}^{(j, m)}$ according to (45)-(47).

c) Calculate $\eta_{t}^{(j, m)}$ and $\Lambda_{t}^{(j, m)}$ according to (50)

d) Calculate the non-normalized weight $\tilde{w}_{t \mid T}^{(j, m)} a c$ cording to (30) e) Sample $u \sim \mathcal{U}[0,1]$

f) If $\tilde{w}_{t \mid T}^{(j, m)} /\left(w_{t \mid t}^{(j)} \rho\right) \geq u$

i) Set $\tilde{x}_{t \mid T}^{n,(m)}=\tilde{x}_{t \mid t}^{n,(j)}$ and $w_{t \mid T}^{(m)}=1 / M_{S}$

ii) Update $\hat{\Omega}_{t}^{(m)}$ and $\hat{\lambda}_{t}^{(m)}$ using (48)

iii) Calculate the conditionally linear states and their covariances

$$
\begin{aligned}
P_{t \mid T}^{(m)} & =\left(\left(P_{t \mid t}^{(j)}\right)^{-1}+\Omega_{t}^{(m)}\right)^{-1} \\
\tilde{x}_{t \mid T}^{l,(m)} & =P_{t \mid T}^{(m)}\left(\left(P_{t \mid t}^{(j)}\right)^{-1} \tilde{x}_{t \mid t}^{l,(j)}+\lambda_{t}^{(m)}\right)
\end{aligned}
$$

iv) Set $L \leftarrow L \backslash m$

g) Set $k \leftarrow k+1$

8) If $L$ is not empty, then, for each $m \in L$

a) For each $n \in\left\{1, \ldots, M_{F}\right\}$

i) Calculate $Z_{t}^{(n, m)}, \Omega_{t}^{(n, m)}$, and $\lambda_{t}^{(n, m)}$ according to (45)-(47).

ii) Calculate $\eta_{t}^{(n, m)}$ and $\Lambda_{t}^{(n, m)}$ according to (50)

iii) Calculate the non-normalized weight $\tilde{w}_{t \mid T}^{(n, m)}$ according to (30)

b) Normalize the smoothed weights

$$
w_{t \mid T}^{(n, m)}=\frac{\tilde{w}_{t \mid T}^{(n, m)}}{\Sigma_{n=1}^{M_{F}} \tilde{w}_{t \mid T}^{(n, m)}}
$$

c) Draw $j \sim \mathcal{C}\left(\left\{w_{t \mid T}^{(n, m)}\right\}_{n=1}^{M_{F}}\right)$

d) Set $\tilde{x}_{t \mid T}^{n,(m)}=\tilde{x}_{t \mid t}^{n,(j)}, w_{t \mid T}^{(m)}=1 / M_{S}, \Omega_{t}^{(m)}=$ $\Omega_{t}^{(j, m)}$, and $\lambda_{t}^{(m)}=\lambda_{t}^{(j, m)}$

e) Update $\hat{\Omega}_{t}^{(m)}$ and $\hat{\lambda}_{t}^{(m)}$ using (48)

f) Calculate the conditionally linear states and their covariances

$$
\begin{aligned}
P_{t \mid T}^{(m)} & =\left(\left(P_{t \mid t}^{(j)}\right)^{-1}+\Omega_{t}^{(m)}\right)^{-1} \\
\tilde{x}_{t \mid T}^{l,(m)} & =P_{t \mid T}^{(m)}\left(\left(P_{t \mid t}^{(j)}\right)^{-1} \tilde{x}_{t \mid t}^{l,(j)}+\lambda_{t}^{(m)}\right)
\end{aligned}
$$

9) Return to step 3 if $t>1$, terminate otherwise

Note that in Algorithm 2, $\rho$ is the upper bound for the nonnormalized particle weight, such that

$$
\frac{\tilde{w}_{t \mid T}^{(j, m)}}{w_{t \mid t}^{(j)}}<\rho .
$$

The RB-PF used in Step 1) in Algorithm 2 is shown in Alogirthm 3. It is based on model 4 and Algorithm 1 in [24]. By using the model structure (21) it can be greatly simplified and the shown algorithm is obtained.

Algorithm 3 (Rao-Blackwellized SIR Particle Filter).

1) Set $t \leftarrow 1$ and initialize

- the nonlinear states as $\tilde{x}_{1 \mid 1}^{n,(m)} \sim p\left(x_{1}^{n}\right)$ and the particle weights as $w_{0 \mid 0}^{(m)}=1 / M_{F}$,

- the prediction of the linear states as $\tilde{x}_{1 \mid 1}^{l,(m)}=\mu_{x_{1}^{l}}$,

- the predicted covariance of the linear states as $P_{1 \mid 1}=C_{x_{1}^{l}}$

for $m=1, \ldots, M_{F}$. 
2) Calculate the importance weight

$$
\tilde{w}^{(m)}=w_{t-1 \mid t-1}^{(m)} p\left(y_{t} \mid \tilde{x}_{t \mid t}^{(m)}\right)
$$

3) Normalize the importance weights

$$
w_{t \mid t}^{(m)}=\frac{\tilde{w}^{(m)}}{\sum_{m=1}^{M_{F}} \tilde{w}^{m}} .
$$

4) If $\left(\sum_{i=1}^{M_{F}}\left(w_{t \mid t}^{(m)}\right)^{2}\right)^{-1} \leq M_{T}$ resample with replacement such that

$$
P\left(x_{t}=\tilde{x}_{t \mid t}^{(m)}\right)=w_{t \mid t}^{(m)}
$$

and set $w_{t \mid t}^{(m)}=1 / M_{F}$.

5) Jitter the particles

$$
\bar{x}_{t \mid t}^{(m)}=\tilde{x}_{t \mid t}^{(m)}+c
$$

with

$$
c \sim \mathcal{N}(0, \Sigma)
$$

according to (36).

6) Predict $M_{F}$ new particles for the non-linear states

$$
\tilde{x}_{t+1 \mid t}^{n,(m)} \sim p\left(x_{t+1}^{n} \mid \bar{x}_{t \mid t}^{n,(m)}, \tilde{x}_{t \mid t}^{l,(m)}\right)
$$

7) Calculate the linear states given $\tilde{x}_{t+1 \mid t}^{n,(m)}$ using the Kalman filter prediction

$$
\begin{aligned}
\tilde{x}_{t+1 \mid t+1}^{l,(m)}= & \bar{A}^{l} \tilde{x}_{t \mid t}^{l,(m)}+\left(Q^{n l}\right)^{T}\left(\bar{Q}^{n}\right)^{-1} z \\
& +L\left(z-A^{n} x_{t \mid t}^{l,(m)}\right)
\end{aligned}
$$

and

$$
P_{t+1 \mid t+1}=\bar{A}^{l} P_{t \mid t}\left(\bar{A}^{l}\right)^{T}-L N L^{\top}
$$

where

$$
\begin{aligned}
\bar{Q}^{n} & =Q^{n}+\Sigma \\
\bar{A}^{l} & =A^{l}-\left(Q^{n l}\right)^{T}\left(\bar{Q}^{n}\right)^{-1} A^{n} \\
\bar{Q}^{l} & =Q^{l}-\left(Q^{n l}\right)^{T}\left(\bar{Q}^{n}\right)^{-1} Q^{n l} \\
z^{(m)} & =\tilde{x}_{t+1 \mid t}^{n,(m)}-F^{n} \tilde{x}_{t \mid t}^{n,(m)}
\end{aligned}
$$

and

$$
\begin{aligned}
N & =A^{n} P_{t \mid t}\left(A^{n}\right)^{T}+\bar{Q}^{n} \\
L & =\bar{A}^{l} P_{t \mid t}\left(A^{n}\right)^{-1} N^{-1} .
\end{aligned}
$$

8) Set $t \leftarrow t+1$. If $t<T$ return to step 2, otherwise terminate.

It is worth to point out that by jittering the particles in Step 5 in Algorithm 3, we mitigate sample impoverishment [27]. The diagonal jittering covariance matrix $\Sigma$ is chosen such that the $i$-th entry on the diagonal is

$$
[\Sigma]_{i}=\left(K E_{i} M_{F}^{-1 / N}\right)^{2}
$$

and all the off-diagonal entries are zero. In (36) $N$ is the state dimension, $E_{i}$ is the distance between the largest and the smallest value of the particles for the $i$-th state, and $K$ is a user chosen constant controlling the amount of jitter. Since we use a Rao-Blackwellized particle filter, the jittering is only applied to the nonlinear states. This has to be taken into account when updating the linear states in Step 6 as follows. Instead of using the transition density (22b), the covariance matrix of the nonlinear states $Q_{t}^{n}$ becomes

$$
Q_{t}^{n}=B^{n} Q\left(B^{n}\right)^{T}+\Sigma .
$$

Furthermore, the linear states are completely given by (34) since they do not enter the measurement equation. Also, since neither the noise covariance nor the system matrices $\left(A^{n}, A^{l}\right)$ depend on the nonlinear states $x_{t}^{n}$, there is only one covariance matrix $P_{t \mid t}$ (but there still is a complete set of $M_{F}$ linear state vectors $\left.\tilde{x}_{t \mid t}^{l,(m)}\right)$.

\section{Parameter Estimation}

The estimate for the parameters $\theta$ is updated in Step 3 in Algorithm 1. This involves maximizing the expected $\log$-likelihood $\hat{Q}_{M}\left(\theta, \hat{\theta}_{k}\right)$ with respect to $\theta$. First, note that neither (25a) nor (25b) depend on $\theta$ and hence, maximizing $\hat{Q}_{M}\left(\theta, \hat{\theta}_{k}\right)$ is equivalent to maximizing $\hat{J}_{3}$. Also, since a backward simulation particle smoother was used, all particle weights are $w_{t \mid T}^{(m)}=1 / M_{S}$. Finally, note that $y_{a, t}$ and $y_{m, t}$ are independent, see (22b).

Then, the stationary points of $\hat{Q}_{M}$ are found by setting the derivative equal to zero

$$
\begin{aligned}
\frac{\partial \hat{Q}_{M}}{\partial \theta}= & \frac{\partial \hat{J}_{3}}{\partial \theta} \\
= & \frac{1}{M_{S}} \sum_{t=1}^{T} \sum_{m=1}^{M} \frac{\partial \log \left(p\left(y_{t} \mid \tilde{x}_{t \mid T}^{(m)} ; \theta\right)\right)}{\partial \theta} \\
=\frac{1}{M_{S}} \sum_{t=1}^{T} \sum_{m=1}^{M}\left(\frac{\partial \log \left(p\left(y_{a, t} \mid \tilde{x}_{t \mid T}^{(m)} ; \theta\right)\right)}{\partial \theta}\right. & \left.+\frac{\partial \log \left(p\left(y_{m, t} \mid \tilde{x}_{t \mid T}^{(m)} ; \theta\right)\right)}{\partial \theta}\right) \\
=0 &
\end{aligned}
$$

where it was made use of the fact that $\hat{J}_{1}$ and $\hat{J}_{2}$ are independent of $\theta$ in the first equality.

1) Accelerometer: The parameters $\kappa, \eta$, and $l$ enter the problem through the measurement equation for $y_{a, t}$. Hence, the derivative with respect to these parameters is zero for $p\left(y_{m, t} \mid \tilde{x}_{t \mid T}^{(m)} ; \theta\right)$. For the accelerometer log-likelihood, we have 
that

$$
\begin{aligned}
& \frac{\partial \log \left(p\left(y_{a, t} \mid \tilde{x}_{t \mid T}^{(m)} ; \theta\right)\right)}{\partial \theta_{j}} \frac{\partial \log \left(\mathcal{N}\left(y_{a, t} ; 0, h_{a}\left(\tilde{x}_{t \mid T}^{(m)} ; \theta\right)^{2}+\sigma_{a}^{2}\right)\right)}{\partial \theta_{j}} \\
&= \frac{\partial}{\partial \theta_{j}}\left(-\frac{1}{2} \log (2 \pi)-\frac{1}{2} \log \left(h_{a}\left(\tilde{x}_{t \mid T}^{(m)} ; \theta\right)^{2}+\sigma_{a}^{2}\right)\right. \\
&\left.-\frac{y_{a, t}^{2}}{2\left(h_{a}\left(\tilde{x}_{t \mid T}^{(m)} ; \theta\right)^{2}+\sigma_{a}^{2}\right)}\right) \\
&=-\frac{h_{a}\left(\tilde{x}_{t \mid T}^{(m)} ; \theta\right)}{\left(h_{a}\left(\tilde{x}_{t \mid T}^{(m)} ; \theta\right)^{2}+\sigma_{a}^{2}\right)^{2}}\left(h_{a}\left(\tilde{x}_{t \mid T}^{(m)} ; \theta\right)^{2}+\sigma_{A}^{2}-y_{a, t}^{2}\right) \\
& \times \frac{\partial h_{a}\left(\tilde{x}_{t \mid T}^{(m)} ; \theta\right)}{\partial \theta_{j}}
\end{aligned}
$$

which gives for $\partial \hat{J}_{3} / \partial \theta_{j}$

$$
\begin{aligned}
\frac{\partial \hat{J}_{3}}{\partial \theta_{j}}=-\frac{1}{M_{S}} & \sum_{t=1}^{T} \sum_{m=1}^{M} \frac{h_{a}\left(\tilde{x}_{t \mid T}^{(m)} ; \theta\right)}{\left(h_{a}\left(\tilde{x}_{t \mid T}^{(m)} ; \theta\right)^{2}+\sigma_{a}^{2}\right)^{2}} \\
& \times\left(h_{a}\left(\tilde{x}_{t \mid T}^{(m)} ; \theta\right)^{2}+\sigma_{A}^{2}-y_{a, t}^{2}\right) \frac{\partial h_{a}\left(\tilde{x}_{t \mid T}^{(m)} ; \theta\right)}{\partial \theta_{j}}
\end{aligned}
$$

The measurement function $h_{a}\left(\tilde{x}_{t \mid T}^{(m)} ; \theta\right)$ is defined in (12) and it is apparent that (40) does not exhibit a closed form solution for any of $\kappa, \eta$, or $l$. Hence, numerical methods have to be employed in this case. To this end, the Newton-Raphson method is used in this paper.

2) Magnetometer: The magnetic moment $m$ only appears in the measurement equation of $y_{m, t}$. First, note that the mean of $p\left(y_{m, t} \mid \tilde{x}_{t \mid T}^{(m)} ; \theta\right)$ can be rewritten as

$$
h_{m}\left(\tilde{x}_{t \mid T}^{(m)} ; \theta\right)=\underbrace{\frac{3\left(r_{t} r_{t}^{T}\right)-\left|r_{t}\right|^{2} I_{3}}{\left|r_{t}\right|^{5}}}_{\triangleq H_{m}\left(\tilde{x}_{t \mid T}^{(m)}\right)} m
$$

where $I_{3}$ is the $3 \times 3$ identity matrix. Equation (41) shows that the model is linear in $m$ and hence, the derivative of the $\log$-likelihood with respect to $m$ is [40]

$$
\begin{aligned}
\frac{\partial \log \left(p\left(y_{m, t} \mid \tilde{x}_{t \mid T}^{(m)} ; \theta\right)\right)}{\partial m}= & H_{m}\left(\tilde{x}_{t \mid T}^{(m)}\right)^{T} C_{m}^{-1} H_{m}\left(\tilde{x}_{t \mid T}^{(m)}\right) m \\
& -H_{m}\left(\tilde{x}_{t \mid T}^{(m)}\right)^{T} C_{m}^{-1} y_{m, t}
\end{aligned}
$$

and the derivative of $\hat{J}_{3}$ with respect to $m$ becomes

$$
\begin{aligned}
\frac{\partial \hat{J}_{3}}{\partial m}= & \frac{1}{M_{S}} \sum_{t=1}^{T} \sum_{m=1}^{M}\left(H_{m}\left(\tilde{x}_{t \mid T}^{(m)}\right)^{T} C_{m}^{-1} H_{m}\left(\tilde{x}_{t \mid T}^{(m)}\right) m\right. \\
& \left.-H_{m}\left(\tilde{x}_{t \mid T}^{(m)}\right)^{T} C_{m}^{-1} y_{m, t}\right) \\
= & \frac{1}{M_{S}} \sum_{t=1}^{T} \sum_{m=1}^{M} H_{m}\left(\tilde{x}_{t \mid T}^{(m)}\right)^{T} C_{m}^{-1} H_{m}\left(\tilde{x}_{t \mid T}^{(m)}\right) m \\
& -\frac{1}{M_{S}} \sum_{t=1}^{T} \sum_{m=1}^{M} H_{m}\left(\tilde{x}_{t \mid T}^{(m)}\right)^{T} C_{m}^{-1} y_{m, t}
\end{aligned}
$$

Setting (43) to zero leads to the closed form estimator for $m$ given by

$$
\begin{aligned}
\hat{m}= & \left(\sum_{t=1}^{T} \sum_{m=1}^{M} H_{m}\left(\tilde{x}_{t \mid T}^{(m)}\right)^{T} C_{m}^{-1} H_{m}\left(\tilde{x}_{t \mid T}^{(m)}\right)\right)^{-1} \\
& \times\left(\sum_{t=1}^{T} \sum_{m=1}^{M} H_{m}\left(\tilde{x}_{t \mid T}^{(m)}\right)^{T} C_{m}^{-1} y_{m, t}\right) .
\end{aligned}
$$

\section{Simulations}

In order to verify the proposed method under controlled conditions and to understand the limitations, it is simulated first.

\section{A. Setup}

The simulation setup is as follows. A car passing the sensor starting at

$$
x_{1}=\left[\begin{array}{llll}
-5 & 2.5 & 20 & 0.2
\end{array}\right]^{T}
$$

with process noise covariance

$$
Q=\left[\begin{array}{cccc}
5 & 0 & 0 & 0 \\
0 & 0.1 & 0 & 0 \\
0 & 0 & 5 & 0 \\
0 & 0 & 0 & 0.1
\end{array}\right]
$$

is simulated. The parameters of the measurement equations are chosen as

$$
\begin{gathered}
m=\left[\begin{array}{ccc}
2 & -2 & 2
\end{array}\right]^{T}, \\
\kappa=10, \\
\eta=2,
\end{gathered}
$$

and

$$
l=2.7 \mathrm{~m} .
$$

The measurement noise (co-)variances were chosen as

$$
C_{m}=5 \times 10^{-5} I_{3}
$$

and

$$
\sigma_{a}^{2}=1 \times 10^{-5} .
$$

In total, $T=1,000$ samples are simulated which corresponds to a complete passage of the car. The measurement signals generated by this setup are shown in Fig. 2. Fig. 2a shows the signals for the magnetometer and Fig. $2 b$ depicts the vibrations. 


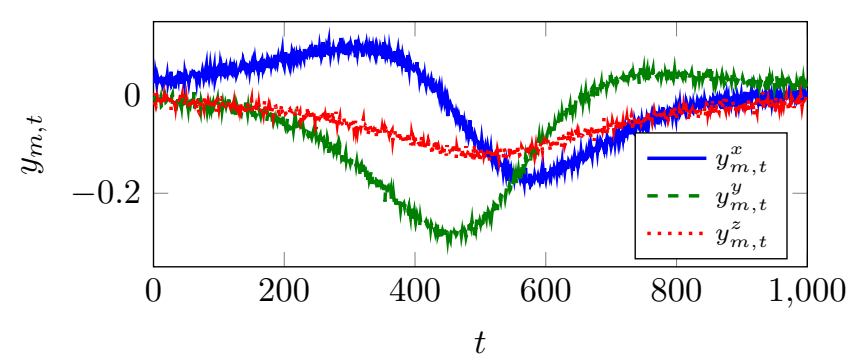

(a)

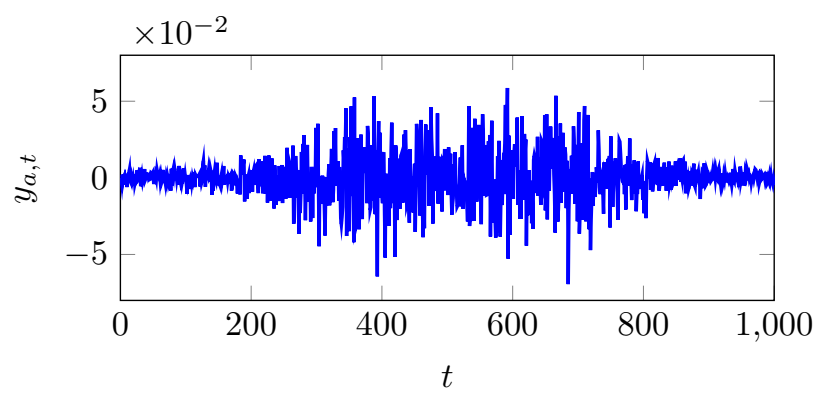

(b)

Fig. 2. Simulated measurement signals. (a) Magnetometer signals and (b) accelerometer signal.

For the particle smoother, the mean and covariance of the initial distribution $p\left(x_{1}\right)$ are chosen as

$$
\mu_{x_{1}}=\left[\begin{array}{llll}
-6 & 2 & 25 & 0
\end{array}\right]^{T}
$$

and

$$
C_{x_{1}}=\left[\begin{array}{cccc}
20 & 0 & 0 & 0 \\
0 & 1 & 0 & 0 \\
0 & 0 & 100 & 0 \\
0 & 0 & 0 & 1
\end{array}\right] .
$$

$M_{F}=1,000$ and $M_{S}=100$ particles were used in the particle filter and smoother, respectively. This is expected to yield good performance at a reasonable computing cost. The initial value for the parameters $\theta_{0}$ is chosen randomly within $\pm 25 \%$ of the true parameter value. Finally, a total of 20 Monte Carlo simulations were run in order to verify the statistical properties.

We also compare the proposed solution to the same expectation maximization approach but where the joint smoothing density is approximated as a multivariate Gaussian distribution by using an unscented Rauch-Tung-Striebel smoother (URTSS) as introduced in [28] and [29]. This is to see whether the computationally more intense method of using the particle smoother yields better estimates than the approximation using the URTSS. This comparison is chosen since it allows for easily comparing the results of the two methods.

\section{B. Results}

1) States: The mean position estimation errors (solid) together with the $2 \sigma$-bounds (dashed) of the 20 simulations are shown in Fig. 3 for the longitudinal $\left(r_{t}^{x}\right.$, top) and lateral $\left(r_{t}^{y}\right.$, bottom) positions, respectively. Both errors converge quickly to zero and the confidence interval narrows as more and more data is obtained. However, the bounds increase again towards

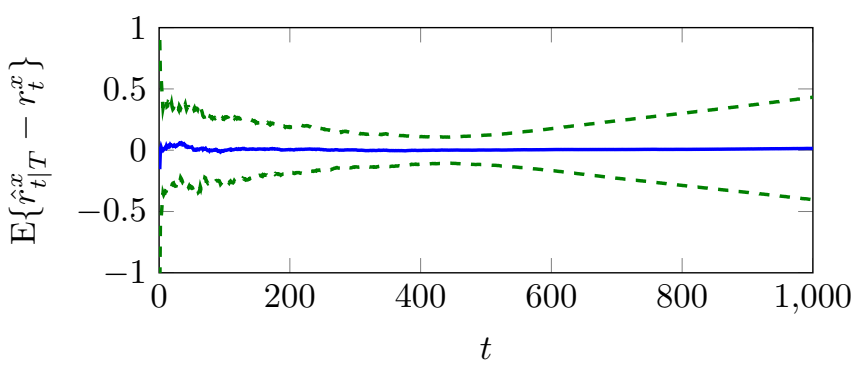

(a)

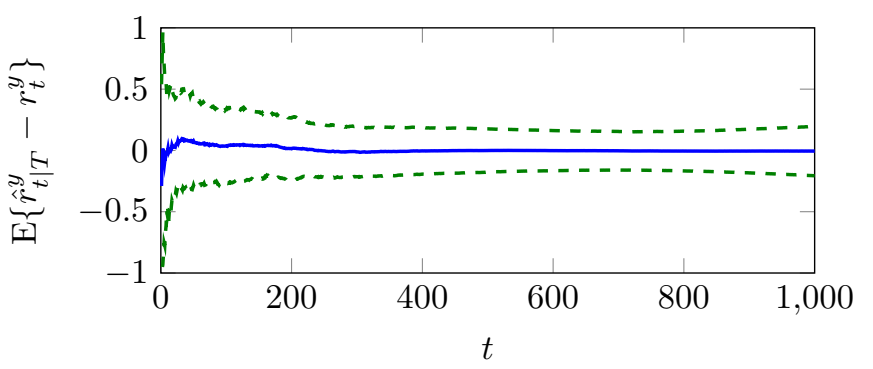

(b)

Fig. 3. Mean state estimation error of the $20 \mathrm{MC}$ simulations (solid) and $2 \sigma$-bounds (dashed) for (a) x-position and (b) y-position.

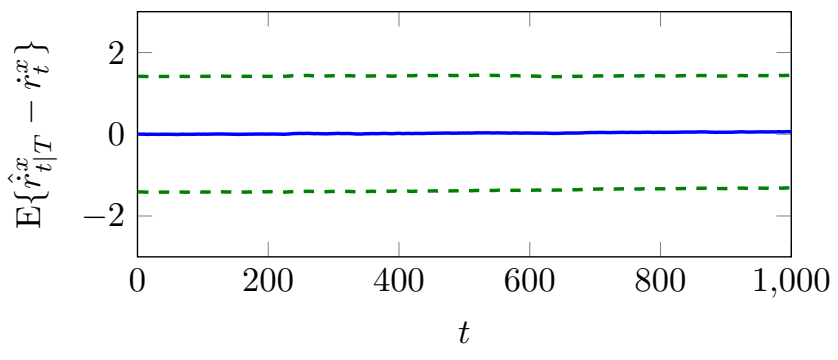

(a)

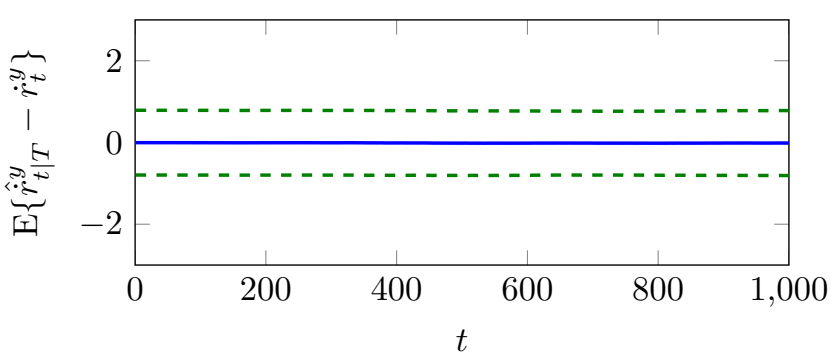

(b)

Fig. 4. Mean state estimation error of the $20 \mathrm{MC}$ simulations (solid) and $2 \sigma$-bounds (dashed) for (a) $\mathrm{x}$-speed and (b) $\mathrm{y}$-speed.

the end of the passage. This is especially pronounced for the lateral position which enters the measurement equation as $\left|r_{t}\right|=\sqrt{\left(r_{t}^{x}\right)^{2}+\left(r_{t}^{y}\right)^{2}}$ and once $\left|r_{t}^{x}\right| \gg\left|r_{t}^{y}\right|$ we have that $\left|r_{t}\right| \approx\left|r_{t}^{x}\right|$.

Fig. 4 shows the mean estimation error (solid) for the longitudinal ( $\dot{r}_{t}^{x}$, top) and lateral $\left(\dot{r}_{t}^{y}\right.$, bottom) speeds. Again, the $2 \sigma$ bounds are included (dashed). Since the speeds correspond to the unmeasured, linear states and are calculated conditioned on the nonlinear states (the vehicle position) these also converge quickly as the position error converges to zero. 
TABLE I

TRUE AND MEAN OF THE ESTIMATED PARAMETER VALUES TOGETHER WITH THEIR STANDARD DEVIATIONS IN PARENTHESES FOR THE PROPOSED PARTICLE SMOOTHER (PS) AND THE UNSCENTED RAUCH-TUNG-STRIEBEL SMOOTHER (URTSS).

\begin{tabular}{lccc}
\hline Parameter & True $\theta$ & PS & URTSS \\
\hline \multirow{4}{m}{} & {$\left[\begin{array}{c}2 \\
-2 \\
2\end{array}\right]$} & {$\left[\begin{array}{c}2 \\
-2.03 \\
1.98\end{array}\right]\left(\left[\begin{array}{l}0.25 \\
0.27 \\
0.29\end{array}\right]\right)$} & {$\left[\begin{array}{c}1.99 \\
-2.05 \\
1.93\end{array}\right]\left(\left[\begin{array}{c}0.3 \\
0.29 \\
0.31\end{array}\right]\right)$} \\
$\kappa$ & 10 & $10.78(1.97)$ & $10.32(1.44)$ \\
$\eta$ & 2 & $2.05(0.24)$ & $2.02(0.26)$ \\
$l$ & 2.7 & $2.97(0.47)$ & $2.75(0.4)$ \\
\hline
\end{tabular}

2) Parameters: The mean of the estimated parameters together with their standard deviations are shown in Table I. It can be seen that unbiased estimations with fairly low variance are obtained for the magnetic moment $m$. However, it appears that the parameters related to the accelerometer measurement are more difficult to estimate, probably due to the randomness of the signal. The wheelbase $l$ is biased and the estimate for $\kappa$ exhibits a relatively large standard deviation.

3) Comparison: The results of the comparison between the particle smoother approximation and the unscented RauchTung-Striebel smoother (URTSS) are shown in Table I and Fig. 5. As it can be seen from the table, the parameters estimated by using the URTSS are almost equally good, both in terms of biasedness and standard deviation.

In Fig. 5, the root mean squared error (RMSE) of the postion estimate $\sqrt{\left(\hat{r}_{t}^{x}-r_{t}^{x}\right)^{2}+\left(\hat{r}_{t}^{y}-r_{t}^{y}\right)^{2}}$ and speed estimate $\sqrt{\left(\hat{\dot{r}}_{t}^{x}-\dot{r}_{t}^{x}\right)^{2}+\left(\hat{\dot{r}}_{t}^{y}-\dot{r}_{t}^{y}\right)^{2}}$ are shown. It can be seen that the estimation using the particle smoother achieves a lower RMSE for both, the position as well as the speed estimate. The difference, however, is not too big.

\section{Discussion}

The simulations indicate that the method is feasible. Good tracking performance was achieved with fast convergence and unbiased position and speed estimates while the target is in front of the sensor.

Regarding the estimation of the unknown parameters in the measurement equations, two conclusions can be drawn. First, the parameters associated with the magnetometer $(m)$ can be estimated bias-free and with high accuracy. Second, the material parameters affecting wave propagation $(\kappa$ and $\eta$ ) and the wheelbase $(l)$ are less precise. The less accurate material parameters as such are not a serious issue since these parameters are of secondary nature. However, the bias in $l$ is an issue since it is important in traffic monitoring applications.

Finally, the comparison of the proposed particle smoother to the unscented Rauch-Tung-Striebel smoother showed that the particle-based method achieves a lower root mean squared error for both, the position as well as the speed estimate. The difference between the two is not too big and thus, if the extra accuracy is not needed, an unscented Rauch-TungStriebel smoother might be sufficient.

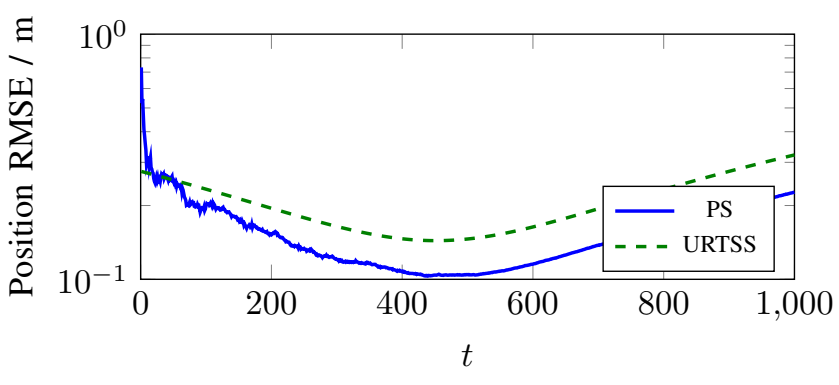

(a)

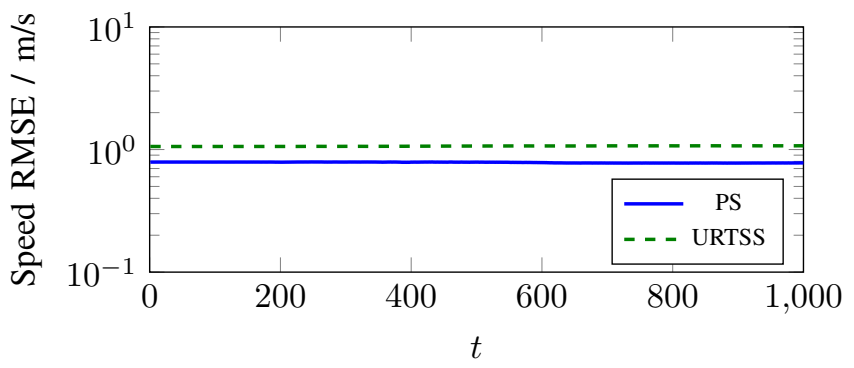

(b)

Fig. 5. Comparison of using the proposed particle smoother (PS; solid) and an unscented Rauch-Tung-Striebel smoother (URTSS; dashed) for approximating the joint smoothing density. RMSE for (a) position and (b) speed.

\section{REsults}

The simulations in Section IV showed that the proposed method is feasible. In order to test the applicability in practice, it was also applied to real measurement data.

\section{A. Measurement Setup}

The measurements were conducted on an arterial road (speed limit $90 \mathrm{~km} / \mathrm{h}$ ) connecting Luleå city to European route E4 during daytime on a spring day. The measurement setup consisted of an accelerometer, a Brüel \& Kjær type 4524 [41], firmly mounted to the road surface at the side of the road. As for the magnetometer, a combination of a 2-axis Honeywell HMC6042 (x- and y-axes) [42] and a Honeywell HMC1051 (z-axis) [43] with an evaluation board was used. A second sensor combination was installed with a separation of $1 \mathrm{~m}$ as a secondary measurement. All sensors were connected to a data recorder where the measurement signals were sampled at $f_{s}=16,384 \mathrm{~Hz}$. Finally, the measured signals were downsampled to $f_{s}=2,048 \mathrm{~Hz}$ and offsets and drift were removed before processing. A total of 50 minutes of measurement data was acquired using this measurement setup.

An example of the measured signals for a passenger car passing the sensor is depicted in Fig. 6. It can be seen that both the measured magnetic disturbance as well as the measured vibration can be compared to the simulated scenario in Section IV. However, the attenuation of the vibrations seems to be less which results in a broader pulse (see Fig. $2 \mathrm{~b}$ and Fig. 6b).

\section{B. Results}

1) States: Fig. 7 shows the average position estimate for both the longitudinal $(\mathrm{x})$ and lateral $(\mathrm{y})$ positions. The $\mathrm{x}$ - 


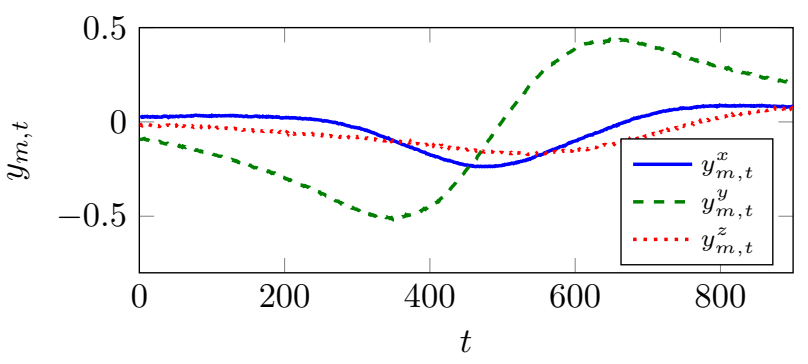

(a)

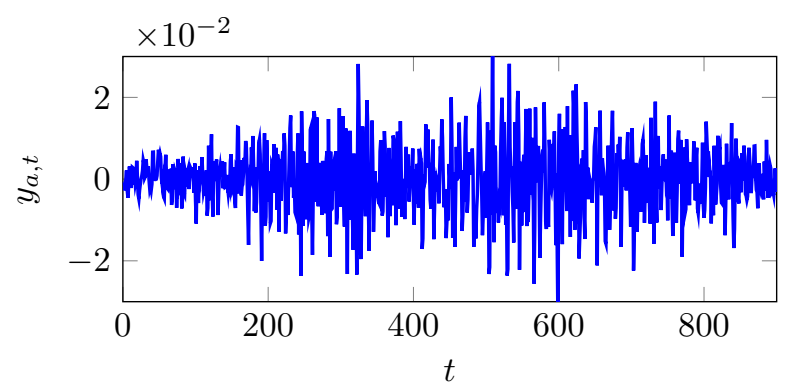

(b)

Fig. 6. Real measurement signals for the illustrated case. (a) Magnetometer and (b) accelerometer.

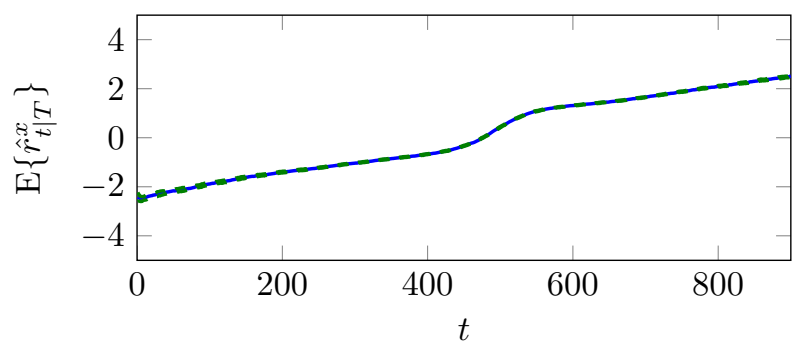

(a)

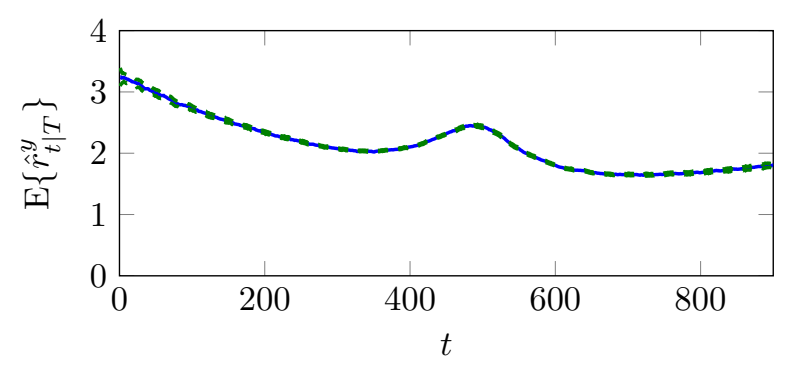

(b)

Fig. 7. Mean state estimation (solid) and $2 \sigma$ bounds (dashed) for (a) longitudinal position and (b) lateral position.

position follows a relatively smooth trend starting around $-3 \mathrm{~m}$ and ending around $3 \mathrm{~m}$ with a slight variation around $t=500$ (Fig. 7a). The y-position on the other hand starts around $4 \mathrm{~m}$ and decreases to about $2 \mathrm{~m}$ (Fig. 7b). Furthermore, a similar smooth variation is observed for the longitudinal position. In practice, this means that the vehicle actually approaches the side of the road during the passage.

The mean estimated lateral and longitudinal speeds are shown in Fig. 8. It can be observed that both speeds are estimated to be approximately constant. The $\mathrm{x}$-speed is around

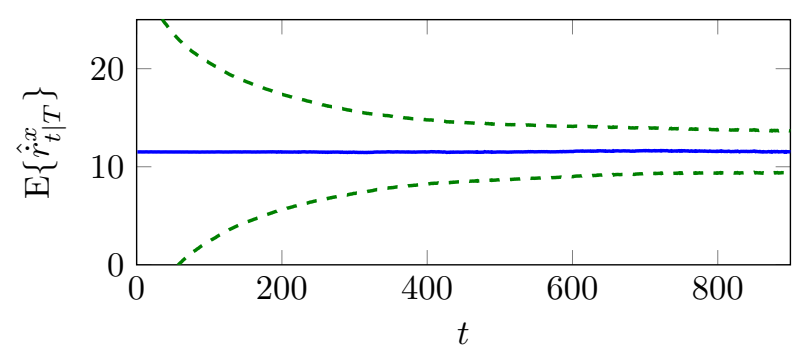

(a)

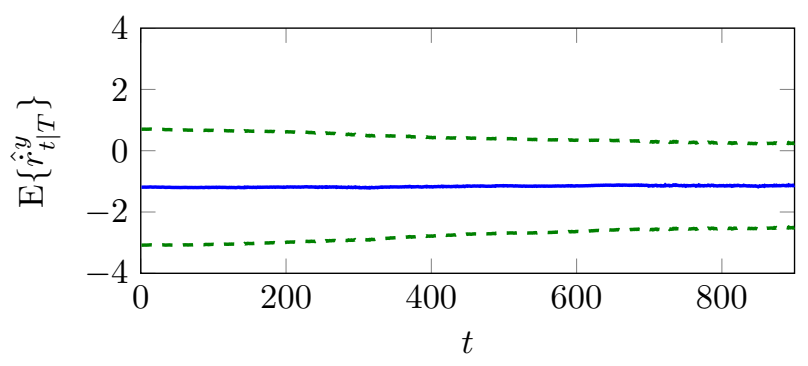

(b)

Fig. 8. Mean state estimation (solid) and $2 \sigma$ bounds (dashed) for (a) longitudinal speed and (b) lateral speed.

$12 \mathrm{~m} / \mathrm{s}$ which significantly deviates from the reference speed of $20.8 \mathrm{~m} / \mathrm{s}$ (estimated using cross-correlation of the second sensor pair). However, it corresponds well to the total distance covered in x-direction during the passage (see Fig. 7a). Furthermore, the $y$-speed lies around $-1 \mathrm{~m} / \mathrm{s}$ which again corresponds to the vehicle slightly moving towards the road side.

2) Parameters: The estimated parameters for the illustrated case are as follows. The magnetic moment was estimated to be

$$
\hat{m}=\left[\begin{array}{c}
3.72 \\
-0.93 \\
1.24
\end{array}\right] .
$$

For the accelerometer, the material parameters were estimated to be

$$
\hat{\kappa}=0.83
$$

and

$$
\hat{\eta}=1.77
$$

Furthermore, the wheelbase was estimated to be

$$
\hat{l}=2.54 \text {. }
$$

\section{Discussion}

For the estimated vehicle trajectory, the results presented seem reasonable but biased. Both the estimated position and speed seem to underestimate the actual motion of the vehicle as suggested by the reference measurement using the secondary sensor. Furthermore, the rather large variation in the position estimate (Fig. 7) around $t=500$ seems unreasonable. This suggest that there are other effects affecting the state estimates. A reason could be undermodeling of the measurements which leads to unexplained data which in turn is absorbed into the state estimate. 
Furthermore, the lateral motion of the vehicle is probably not very accurate. Considering that the road imposes large constraints on the possible motion of the vehicle, the motion model could probably be simplified in order to obtain a better estimation, for example by assuming longitudinal motion only and considering the lateral position as a fixed parameter instead.

It is difficult to conclusively discuss the estimated parameters since there are no true reference values available for comparison. The results for these seem reasonable with the magnetic moment in the range one would expect it (see, for example [10]) and the wheelbase around the average for passenger cars. These results should be verified more thoroughly in the future.

\section{CONCLusions}

In this article, it was shown how particle system identification consisting of a Rao-Blackwellized particle smoother using a low number of particles and the expectation maximization algorithm can be applied for estimating the vehicle trajectory as well as unknown, static parameters.

The simulation results showed that the method is feasible and good results for both, the trajectory as well as the parameters can be obtained. Applied to real measurement data the performance degraded, probably due to model uncertainties and the range of the true parameters that complicate the problem.

Clearly, due to the iterative nature of the algorithm, the method is not feasible for real-time applications. However, in practice it could be used to auto-tune a traffic monitoring system to initially determine the unknown parameters and then a conventional (Rao-Blackwellized) particle filter could be used for subsequent tracking applications.

\section{APPENDIX}

The statisitcs required for calculating the smoothed particle weight and backward propagation in (30) and Algorithm 2 stem from the Rao-Blackwellization. Their derivation can be found in [26] and the resulting expressions for these quantities are given below. For brevity, $x^{T} C x$ is written as $\|x\|_{C}, f_{t}^{n} \triangleq$ $f^{n}\left(x_{t}^{n}\right)$, and analogously for $A_{t}^{n}, f_{t}^{l}$, and $A_{t}^{l}$. Furthermore, $Q_{t}^{n} \triangleq \operatorname{Cov}\left\{x_{t}^{n}, x_{t}^{n}\right\}$ is the covariance of the nonlinear states, $Q_{t}^{l} \triangleq \operatorname{Cov}\left\{x_{t}^{l}, x_{t}^{l}\right\}$, and $Q_{t}^{n l} \triangleq \operatorname{Cov}\left\{x_{t}^{n}, x_{t}^{l}\right\}$.

\section{A. Backward Prediction}

The statistics $Z_{t}, \Omega_{t}$, and $\lambda_{t}$ are given by

$$
\begin{aligned}
Z_{t}= & \left|M_{t}\right|^{-1 / 2}\left|Q_{t}^{n}\right|^{-1 / 2} \exp \left(-\frac{1}{2} \tau_{t}\right), \\
\Omega_{t}= & \left(\bar{A}_{t}^{l}\right)^{T}\left(\hat{\Omega}_{t+1}-\hat{\Omega}_{t+1} \bar{\Gamma} M_{t}^{-1} \bar{\Gamma}^{T} \hat{\Omega}_{t+1}\right) \bar{A}_{t}^{l} \\
& +\left(A_{t}^{n}\right)^{T}\left(Q_{t}^{n}\right)^{-1} A_{t}^{n}, \\
\lambda_{t}= & \left(\bar{A}_{t}^{l}\right)^{T}\left(I_{N_{l}}-\hat{\Omega}_{t+1} \bar{\Gamma} M_{t}^{-1} \bar{\Gamma}^{T}\right) m_{t} \\
& +\left(A_{t}^{n}\right)^{T}\left(Q_{t}^{n}\right)^{-1}\left(\tilde{x}_{t+1}-f_{t}^{n}\right) .
\end{aligned}
$$

where

$$
\begin{aligned}
& m_{t}=\hat{\lambda}_{t+1}-\hat{\Omega}_{t+1} \bar{f}_{t}^{l} \\
& M_{t}=\bar{\Gamma}^{T} \hat{\Omega}_{t+1} \bar{\Gamma}+I_{N_{n}}, \\
& \tau_{t}=\left\|\tilde{x}_{t+1}^{n}-f_{t}^{n}\right\|_{\left(Q_{t}^{n}\right)^{-1}}+\left\|\bar{f}_{t}^{l}\right\|_{\hat{\Omega}_{t+1}} \\
& \quad-2\left(\hat{\lambda}_{t+1}\right)^{T} \bar{f}_{t}^{l}-\left\|\bar{\Gamma}^{T} m_{t}\right\|_{M_{t}^{-1}},
\end{aligned}
$$

and $\bar{\Gamma}$ is the Cholesky decomposition (lower triangular) of $\bar{Q}_{t}^{l}$ such that $\bar{Q}_{t}^{l}=\bar{\Gamma}^{T} \bar{\Gamma} . \bar{A}_{t}^{l}, \bar{f}_{t}^{l}$, and $\bar{Q}_{t}^{l}$ are given through the decorrelation of the linear- and nonlinear state dynamics as

$$
\begin{aligned}
\bar{f}_{t}^{l} & =f_{t}^{l}-\left(Q_{t}^{n l}\right)^{T}\left(Q_{t}^{n}\right)^{-1}\left(x_{t+1}^{n}-f_{t}^{n}\right), \\
\bar{A}_{t}^{l} & =A_{t}^{l}-\left(Q_{t}^{n l}\right)^{T}\left(Q_{t}^{n}\right)^{-1} A_{t}^{n}, \\
\bar{Q}_{t}^{l} & =Q_{t}^{l}-\left(Q_{t}^{n l}\right)^{T}\left(Q_{t}^{n}\right)^{-1} Q_{t}^{n l} .
\end{aligned}
$$

\section{B. Backward Update}

The statistics $\hat{\Omega}_{t}$ and $\hat{\lambda}_{t}$ are updated using the new measurements as well as the predicted statistics as

$$
\begin{aligned}
& \hat{\Omega}_{t}=\Omega_{t}+C_{t}^{T} R_{t}^{-1} C_{t} \\
& \hat{\lambda}_{t}=\lambda_{t}+C_{t}^{T} R_{t}^{-1}\left(y_{t}-h_{t}\right) .
\end{aligned}
$$

At time $T$, they are initialized according to

$$
\begin{aligned}
& \hat{\Omega}_{T}=C_{T}^{T} R_{T}^{-1} C_{T} \\
& \hat{\lambda}_{T}=C_{T}^{T} R_{T}^{-1}\left(y_{T}-h_{T}\right) .
\end{aligned}
$$

\section{Particle Weight}

The quantities for calculating the non-normalized particle weight (30) are given by

$$
\begin{aligned}
& \Lambda_{t}=\left(\Gamma_{t \mid t}\right)^{T} \Omega_{t} \Gamma_{t \mid t}+I_{N_{l}}, \\
& \eta_{t}=\left\|\tilde{x}_{t \mid t}^{l}\right\|_{\Omega_{t}}-2 \lambda_{t} \tilde{x}_{t \mid t}^{l}-\left\|\left(\Gamma_{t \mid t}\right)^{T}\left(\lambda_{t}-\Omega_{t} \tilde{x}_{t \mid t}^{l}\right)\right\|_{\Lambda_{t}}
\end{aligned}
$$

where $\Gamma_{t \mid t}$ is the Cholesky decomposition (lower triangular) of $P_{t \mid t}$ such that $P_{t \mid t}=\left(\Gamma_{t \mid t}\right)^{T} \Gamma_{t \mid t}$.

\section{REFERENCES}

[1] R. Hostettler, W. Birk, and M. Lundberg Nordenvaad, "Joint vehicle trajectory and model parameter estimation using road side sensors," Sensors Journal, IEEE, vol. 15, no. 9, pp. 5075-5086, Sept 2015.

[2] Y. Bar-Shalom, X. R. Li, and T. Kirubarajan, Estimation with Applications to Tracking and Navigation: Theory, Algorithms and Software. John Wiley \& Sons, 2004.

[3] X. R. Li and V. P. Jilkov, "Survey of maneuvering target tracking: Part I. Dynamic models," Aerospace and Electronic Systems, IEEE Transactions on, vol. 39, no. 4, pp. 1333-1364, 2003.

[4] —, "Survey of maneuvering target tracking: Part III. Measurement models," Proc. SPIE, vol. 4473, pp. 423-446, 2001.

[5] J. Altmann, "Acoustic and seismic signals of heavy military vehicles for co-operative verification," Journal of Sound and Vibration, vol. 273, no. 4-5, pp. 713-740, June 2004.

[6] R. Hostettler, W. Birk, and M. Lundberg Nordenvaad, "Extended Kalman filter for vehicle tracking using road surface vibration measurements," in Decision and Control (CDC), 2012 IEEE 51st Annual Conference on, Maui, HI, USA, December 2012, pp. 5643-5648. 
[7] W. M. Wynn, "Detection, localization, and characterization of static magnetic dipole sources," in Detection and Identification of Visually Obscured Targets. Taylor and Francis, 1999, pp. 337-374.

[8] M. Rakijas, A. Saglembeni, K. K. Kohnen, and H. C. Gilbert, "Magnetic object tracking based on direct observation of magnetic sensor measurements," US Patent 6269324 , July 31, 2001.

[9] M. Birsan, "Non-linear Kalman filters for tracking a magnetic dipole," Defence R\&D Canada, Dartmouth, NS, Canada, Tech. Rep. DRDCATLANTIC-TM-2003-230, December 2003.

[10] N. Wahlström and F. Gustafsson, "Magnetometer modeling and validation for tracking metallic targets," Signal Processing, IEEE Transactions on, vol. 62, no. 3, pp. 545-556, February 2014.

[11] I. Vallivaara, J. Haverinen, A. Kemppainen, and J. Roning, "Magnetic field-based slam method for solving the localization problem in mobile robot floor-cleaning task," in Advanced Robotics (ICAR), 2011 15th International Conference on, June 2011, pp. 198-203.

[12] M. Frassl, M. Angermann, M. Lichtenstern, P. Robertson, B. J. Julian, and M. Doniec, "Magnetic maps of indoor environments for precise localization of legged and non-legged locomotion," in Intelligent Robots and Systems (IROS), 2013 IEEE/RSJ International Conference on, November 2013, pp. 913-920.

[13] P. Robertson, M. Frassl, M. Angermann, M. Doniec, B. J. Julian, M. Garcia Puyol, M. Khider, M. Lichtenstern, and L. Bruno, "Simultaneous localization and mapping for pedestrians using distortions of the local magnetic field intensity in large indoor environments," in Indoor Positioning and Indoor Navigation (IPIN), 2013 International Conference on, October 2013, pp. 1-10.

[14] M. Kok, N. Wahlström, T. B. Schön, and F. Gustafsson, "Mems-based inertial navigation based on a magnetic field map," in Acoustics, Speech and Signal Processing (ICASSP), 2013 IEEE International Conference on, May 2013, pp. 6466-6470.

[15] S. Särkkä, Bayesian Filtering and Smoothing. Cambridge University Press, 2013

[16] P. M. Djurić, M. F. Bugallo, and J. Miguez, "Density assisted particle filters for state and parameter estimation," in Acoustics, Speech and Signal Processing (ICASSP), 2004 IEEE International Conference on, vol. 2, May 2004

[17] G. Storvik, "Particle filters for state-space models with the presence of unknown static parameters," Signal Processing, IEEE Transactions on, vol. 50, no. 2, pp. 281-289, Feb 2002.

[18] N. G. Polson, J. R. Stroud, and P. Müller, "Practical filtering with sequential parameter learning," Journal of the Royal Statistical Society: Series B (Statistical Methodology), vol. 70, no. 2, pp. 413-428, 2008.

[19] C. Andrieu, A. Doucet, and V. B. Tadic, "On-line parameter estimation in general state-space models," in Decision and Control, 2005 and 2005 European Control Conference. CDC-ECC '05. 44th IEEE Conference on, December 2005, pp. 332-337.

[20] N. Chopin, "A sequential particle filter method for static models," Biometrika, vol. 89, no. 3, pp. 539-552, 2002.

[21] A. Doucet and V. Tadić, "Parameter estimation in general state-space models using particle methods," Annals of the Institute of Statistical Mathematics, vol. 55, no. 2, pp. 409-422, 2003.

[22] S. Gibson, A. Wills, and B. Ninness, "Maximum-likelihood parameter estimation of bilinear systems," Automatic Control, IEEE Transactions on, vol. 50, no. 10, pp. 1581-1596, October 2005.

[23] T. B. Schön, A. Wills, and B. Ninness, "System identification of nonlinear state-space models," Automatica, vol. 47, no. 1, pp. 39-49, January 2011.

[24] T. Schön, F. Gustafsson, and P.-J. Nordlund, "Marginalized particle filters for mixed linear/nonlinear state-space models," Signal Processing, IEEE Transactions on, vol. 53, no. 7, pp. 2279-2289, July 2005.

[25] R. Douc, A. Garivier, E. Moulines, and J. Olsson, "Sequential Monte Carlo smoothing for general state space hidden markov models," The Annals of Applied Probability, vol. 21, no. 6, pp. 2109-2145, December 2011.

[26] F. Lindsten, P. Bunch, S. J. Godsill, and T. B. Schön, "Rao-Blackwellized particle smoothers for mixed linear/nonlinear state-space models," in Acoustics, Speech and Signal Processing (ICASSP), 2013 IEEE International Conference on, Vancouver, BC, Canada, May 2013, pp. 62886292.

[27] N. J. Gordon, D. J. Salmond, and A. F. M. Smith, "Novel approach to nonlinear/non-Gaussian Bayesian state estimation," Radar and Signal Processing, IEE Proceedings F, vol. 140, no. 2, pp. 107-113, April 1993.

[28] S. Särkkä, "Unscented Rauch-Tung-Striebel smoother," Automatic Control, IEEE Transactions on, vol. 53, no. 3, pp. 845-849, April 2008.
[29] S. B. Chitralekha, J. Prakash, H. Raghavan, R. B. Gopaluni, and S. L. Shah, "Comparison of expectation-maximization based parameter estimation using particle filter, unscented and extended kalman filtering techniques," in 15th IFAC Symposium on System Identification, Proceedings of the, Saint-Malo, France, July 2009.

[30] F. Gustafsson, F. Gunnarsson, N. Bergman, U. Forssell, J. Jansson, R. Karlsson, and P.-J. Nordlund, "Particle filters for positioning, navigation, and tracking," Signal Processing, IEEE Transactions on, vol. 50, no. 2, pp. 425-437, February 2002.

[31] R. Hostettler, M. Lundberg Nordenvaad, and W. Birk, "The pavement as a waveguide: Modeling, system identification, and parameter estimation," Instrumentation and Measurement, IEEE Transactions on, vol. 63, no. 8, pp. 2052-2063, 2014.

[32] M. Abramowitz and I. A. Stegun, Handbook of Mathematical Functions. Washington, DC, USA: National Institute of Standards and Technology, 1964.

[33] C. J. Dodds and J. D. Robson, "The description of road surface roughness," Journal of Sound and Vibration, vol. 31, no. 2, pp. 175-183, 1973.

[34] P. Andrén, "Power spectral density approximations of longitudinal road profiles," International Journal of Vehicle Design, vol. 40, no. 1, pp. 2-14, 2006.

[35] B. Bruscella, V. Rouillard, and M. Sek, "Analysis of road surface profiles," Journal of Transportation Engineering, vol. 125, no. 1, pp. 55-59, January 1999.

[36] International Organization for Standardization, ISO 8608: Mechanical Vibration - Road Surface Profiles - Reporting of Measured Data, 1995.

[37] A. Doucet and A. M. Johansen, "A tutorial on particle filtering and smoothing: Fifteen years later," Handbook of Nonlinear Filtering, vol. 12, pp. 656-704, 2009.

[38] H. E. Rauch, C. T. Striebel, and F. Tung, "Maximum likelihood estimates of linear dynamic systems," AIAA Journal, vol. 3, no. 8, pp. 1445-1450, August 1965.

[39] E. Taghavi, F. Lindsten, L. Svensson, and T. B. Schön, "Adaptive stopping for fast particle smoothing," in Acoustics, Speech and Signal Processing (ICASSP), 2013 IEEE International Conference on, Vancouver, BC, Canada, May 2013, pp. 6293-6297.

[40] S. M. Kay, Fundamentals of Statistical Signal Processing: Estimation Theory. Upper Saddle River, NJ, USA: Prentice Hall, 1993.

[41] Brüel \& Kjær, Miniature Triaxial DeltaTron Accelerometers - Types 4524, 4524-B and 4524-B-001, datasheet.

[42] Honeywell, 2-Axis Magnetic Sensor Circuit HMC6042, datasheet.

[43] $-1, \quad 2$ and 3 Axis Magnetic Sensors HMC1051/HMC1052/HMC1053, datasheet.

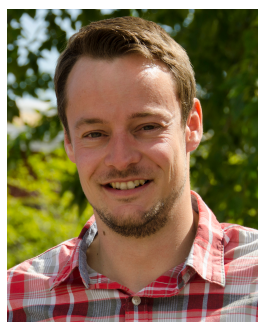

Roland Hostettler (S'10-M'14) received the Dipl. Ing. degree in electrical and communication engineering from Bern University of Applied Sciences, Switzerland in 2007, and the M.Sc. degree in electrical engineering and Ph.D. degree in automatic control from Luleå University of Technology, Sweden in 2009 and 2014, respectively.

$\mathrm{He}$ is currently working as a research associate at Luleå University of Technology, Sweden. His research interests include statistical signal processing with applications to target tracking, intelligent transportation systems, and sensor networks. 


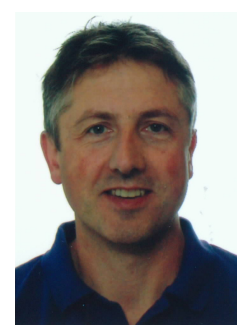

Wolfgang Birk (M'97) is Professor of Automatic Control and Director of Third Cycle Studies at the Department of Computer Science, Electrical and Space Engineering at Luleå University of Technology. He holds a M.Sc. degree in Electrical Engineering from University of Saarland, Germany (1997) and a Ph.D. degree in Automatic Control from Luleå University of Technology (2002).

Birk has a background in the development of Active Safety system of passenger cars, relating to collision avoidance, collision mitigation, and driver state estimation and warning. For his work relating to the development of Volvo Cars Driver Alert, he has received the Henry Ford Technology Award (2007). Birk is co-founder of several spin-off companies from research projects. His research interests are the application of estimation theory, control structure and configuration selection in large scale systems, as well as reconfiguration of control systems. Main application areas are intelligent transport systems and process control.

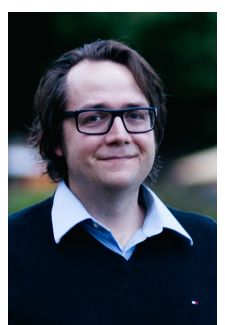

Magnus Lundberg Nordenvaad was born in Luleå, Sweden, in 1973. He received the M.Sc. degree in Computer Engineering from Luleå University of Technology, Luleå, Sweden, and the Ph.D. degree in Signal Processing from Chalmers University of Technology, Gothenburg, Sweden, in 1998 and 2003 respectively. His research interests lie in statistical signal processing and how it applies to sonar, digital communication, radar, process diagnostics among others. He has authored around 60 papers in international journals and conferences.

Dr. Lundberg Nordenvad is currently Deputy Research Director at the Swedish Defence Research Agency (FOI). Previously, he has held faculty positions at The Department of Information Technology, Uppsala University, Uppsala, Sweden and The Department of Computer Science and Electrical Engineering, Luleå University of Technology, Luleå, Sweden. He has also held visiting researcher/professor positions at Purdue University, Colorado State University, and University of Florida. 